\title{
CULTURE SANS ARTIFICE
}

À propos de Dominique Guillo, Les fondements oubliés de la culture. Une approche écologique, Paris, Seuil, 2019, 360 pages

Jérôme Michalon, Antoine Doré

Éditions du Croquant | «Zilsel »

2020/2 N 7 | pages 235 à 260

ISSN 2551-8313

Article disponible en ligne à l'adresse :

https://www.cairn.info/revue-zilsel-2020-2-page-235.htm

Distribution électronique Cairn.info pour Éditions du Croquant.

(C) Éditions du Croquant. Tous droits réservés pour tous pays.

La reproduction ou représentation de cet article, notamment par photocopie, n'est autorisée que dans les limites des conditions générales d'utilisation du site ou, le cas échéant, des conditions générales de la licence souscrite par votre établissement. Toute autre reproduction ou représentation, en tout ou partie, sous quelque forme et de quelque manière que ce soit, est interdite sauf accord préalable et écrit de l'éditeur, en dehors des cas prévus par la législation en vigueur en France. Il est précisé que son stockage dans une base de données est également interdit. 


\title{
Culture sans artifice
}

\author{
Jérôme Michalon ${ }^{1}$ \\ \& Antoine Doré ${ }^{2}$
À propos de
Dominique Guillo,
Les fondements oubliés de la culture.
Une approche écologique, Paris, Seuil, 2019, 360 pages.

Depuis la parution d'un volume consacré aux liens entre chiens et humains ${ }^{3}$, Dominique Guillo, sociologue, directeur de recherche au CNRS, a engagé plusieurs chantiers de recherche ayant trait aux relations anthropozoologiques ${ }^{4}$. Pour ce spécialiste des rapports des sciences sociales aux sciences naturelles, un tel objet fournit l'occasion d'un rapprochement épistémologique entre ces deux ensembles disciplinaires, mais aussi de repenser en profondeur le concept de «culture». Son nouvel essai, Les fondements oubliés de la culture, constitue ainsi la synthèse programmatique - articulée et augmentée - de plus d'une dizaine d’années de recherche. Appelant au développement d'une « histoire naturelle des interactions sociales» (p. 170), D. Guillo consacre la première moitié de l'ouvrage à l'établissement d'un diagnostic épistémologique de l'étude des cultures (humaines et non-humaines) et sa seconde moitié à la présentation et la mise à l'épreuve d'un programme de recherche empirique.

\footnotetext{
1. CNRS, UMR Triangle, Saint Etienne, jerome.michalon@ens-lyon.fr.

2. INRAE, UMR AGIR, Toulouse, antoine.dore@inrae.fr.

3. Dominique Guillo, Des chiens et des humains, Paris, Le Pommier, 2009.

4. Les auteurs souhaitent remercier la rédaction de La Vie des idées et de Zilsel d'avoir accepté la publication de cette recension dans sa version longue : «Àl'école des bêtes», LaViedesldées.fr, 20 novembre 2019, laviedesidees.fr/Dominique-Guillo-Fondements-oublies-culture-ecologique.html. Ils souhaitent également remercier les membres du réseau Imaginaires et Pratiques des Relations AnthropoZoologiques (IPRAZ) pour le travail de lecture collective de l'article Dominique Guillo, «Quelle place faut-il faire aux animaux en sciences sociales? Les limites des réhabilitations récentes de l'agentivité animale », Revue française de sociologie, vol. №56, №1, 2015, p.135-163. Merci donc à : Christophe Baticle, Claire Juliette Camblain, Angela Casal Beytrison, Stéphanie Chanvallon, Léa de Boisseuil, Bénédicte de Villers, Jean Estebanez, Sophie Gallino-Visman, Séverine Lagneaux, Guillaume Marchand, Chloé Mondémé, Sébastien Mouret, Bastien Picard, Patrice Régnier, et Mathieu Sassu. Merci enfin à Marine Maurin. Les propos tenus dans ce texte n'engagent que leurs auteurs.
} 


\section{Le biais identitaire}

Les recherches sur la culture sont prises en charge par deux ensembles de disciplines, qui relèvent soit des sciences humaines et sociales (en particulier l'anthropologie et la sociologie), soit des sciences de la nature (l'écologie comportementale, l'éthologie et la biologie néo-darwinienne). Chacun de ces ensembles étudie la culture à partir de groupes d'animaux (humains et non-humains) appartenant à la même espèce, si bien que la notion de culture semble ne pouvoir émerger que dans des groupes composés d'êtres supposés partager une même identité, du point de vue des chercheurs. Pour D. Guillo, cette approche charrie un tropisme ou un biais identitaire, qui marque à la fois la focale (les relations intraspécifiques ou intragroupes) et les résultats de ces recherches (la culture se déploie uniquement entre entités semblables et accentue leur similitude) ; autrement dit, elle procède (postule) et produit (accentue) de l'identité partagée. Dans un monde où la compréhension de l'interdépendance entre des êtres aussi différents qu'un lombric, une baleine ou une molécule, devient de plus en plus cruciale (D. Guillo souhaite en effet contribuer aux réflexions scientifiques sur l'Anthropocène), ce biais identitaire est un obstacle épistémologique majeur. D’autant plus, selon lui, qu'il est décelable même lorsqu'il s'agit d'analyser les interactions entre humains et animaux, les recherches actuelles insistant davantage sur ce qui rapproche les premiers des seconds que sur ce qui les sépare. Comment donc penser la différence entre les êtres, à la fois comme postulat et comme résultat de leurs interactions? Pour répondre à cette interrogation, l'ouvrage promeut une approche de la culture fondée sur l'étude des ajustements mutuels entre les êtres qui procèdent et produisent de la différence. D. Guillo souhaite donner à cette proposition une valeur programmatique forte et ne pas la cantonner à l'étude des rapports humains-animaux. Il entend bien en effet défendre la possibilité d'étendre ce modèle à toutes les formes d'interactions ${ }^{5}$, y compris entre humains; et donc de revisiter en profondeur les travaux des sciences sociales. Plus généralement, c'est l'adoption d'un nouveau paradigme que vise D. Guillo, dans lequel sciences de la nature et sciences sociales travailleraient conjointement, dans une perspective intégrative.

5. C'est l'auteur lui-même qui met régulièrement l'emphase sur la dimension totalisante de son programme. 


\section{Un diagnostic épistémologique : l'incapacité à penser des cultures interspécifiques}

Cette proposition se déploie par étapes dans le plan de l'ouvrage. Le premier chapitre offre un état, très pédagogique, des recherches sur les sociabilités et les cultures animales en éthologie et en biologie néo-darwinienne. On y rencontre une définition du «social » assez éloignée de celle des sciences du même nom : est social, pour les néo-darwiniens, un comportement qui ne semble pas dépendre des mécanismes de la sélection naturelle (e.g. celui d'individus œuvrant à la survie ou à la perpétuation de gènes dont ils ne sont pas porteurs). Pour les éthologues, en particulier les tenants du concept de «cultures animales », le «social» est plutôt en lien avec les capacités cognitives ou d'apprentissage des animaux. En effet, leur définition de la culture repose sur le «social learning», ou la manière dont des individus se transmettent des comportements, sans que les gènes ne soient directement impliqués. Pour qui penserait encore que l'inné se situerait du côté des animaux et que l'acquis serait un privilège humain, la lecture de ces pages est vraiment à conseiller. Si l'existence de «cultures animales» ne semble plus faire débat, D. Guillo relève tout de même une difficulté, voire une réticence de la part de l'éthologie, à s'intéresser aux rapports entre individus d'espèces différentes. L'étude des «cultures interspécifiques » n'est pas encore à l'ordre du jour, alors même que, depuis plusieurs années, des travaux en sciences sociales portent sur les rapports entre comportements humains et comportements animaux. C'est à ces travaux que l'auteur consacre le second chapitre. Regroupant un ensemble plutôt hétérogène (les animal studies, l'anthropologie de B. Latour, celle de P. Descola, de T. Ingold, l'interactionnisme d'A. Arluke, C. Sanders, ou C. Jerolmack, l'ethnométhodologie de D. Goode, les science studies de D. Haraway, ou E. Crist), D. Guillo reconnaît leur contribution à la connaissance empirique des interactions interspécifiques, mais leur dénie néanmoins toute robustesse épistémologique. Selon D. Guillo, ces travaux dénonceraient unanimement l'existence d'un Grand Partage entre sciences de la nature et sciences de la culture, qui empêcherait de voir les animaux autrement que comme des «machines», et interdirait donc de penser réellement la possibilité de cultures co-construites entre humains et animaux. Cependant, cette thèse du Grand Partage n'est pas historiquement fondée selon lui : les sciences de la nature ne postuleraient pas une frontière aussi hermétique entre 
des humains rationnels, «maîtres et possesseurs de la nature $»$, et des ani maux soumis leur bon vouloir. Au contraire, la dit-il, c'est de ne

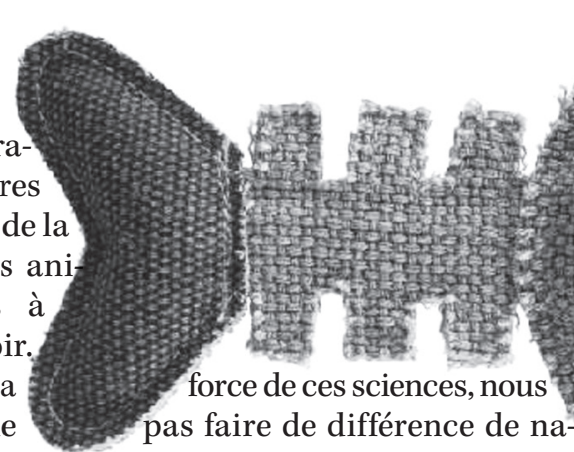
ture entre les hu-mains et les autres animaux, et donc $\mathrm{k}$ de ne plus placer l'humain au centre de toute réflexion ; à l'inverse des sciences sociales qui, par un réflexe constructiviste, ne peuvent être qu'anthropocentrées, même lorsqu'elles affirment vouloir faire le contraire. Ce faisant, les sciences sociales, et a fortiori les travaux cités dans ce chapitre, deviennent largement responsables du cloisonnement ou de l'indifférence réciproque entre des domaines scientifiques qui prennent pour objet des réalités empiriques semblables, et dont les acteurs auraient tout intérêt à discuter. Mais ces deux domaines sont tributaires du tropisme ou biais identitaire, déjà évoqué, et qui fait l'objet du troisième chapitre. L'auteur y détaille un certain nombre d'écueils qui lui sont associés, dont le recours à la notion d'anthropomorphisme. Qu'il soit récusé ou revendiqué, l'anthropomorphisme cristallise les débats autour de la compréhension des comportements animaux. En faisant de l'assimilation des animaux aux humains (ou d'une identité partagée) le critère primordial pour pouvoir parler de vie sociale ou de culture animale, ces débats témoignent de la prégnance du tropisme identitaire.

\section{Un programme empirique : réconcilier l'interaction et l'evolution}

Pour dépasser ces écueils, D. Guillo propose de ne plus aborder les relations humains-animaux avec des concepts trop généraux, mais d'étudier leurs interactions « concrètes et situées ». Dans son quatrième chapitre, il livre ainsi deux exemples d'analyse de ces interactions, issus de son propre travail (un humain qui ouvre une baie vitrée à un chien, et les interactions entre humains et macaques sur un site touristique marocain). Dans ces situations, les différences sont partout : entre les êtres, dans leurs attentes, dans leurs actions, leurs réactions, etc. Et pourtant, des «normes » émergent de ces interactions, qui ne leur préexistaient pas, et qui ne sont pas non plus des «normes communes » (car elles ne concernent pas 
de la même manière les humains et les animaux coprésents lors de l'interaction étudiée, pas plus qu'elles ne concernent tous les humains et tous les animaux). De la différence génère de la différence, donc. Le mystère réside alors dans la multiplication de ces conduites réglées entre humains et animaux, constatées à la fois par les sciences du comportement et les sciences sociales. «Multiplication » ne signifie pas « reproduction à l'identique», puisque c'est précisément l'apprentissage de conduites nouvelles chez les animaux au contact des humains qui constitue le fait notable (et inédit) mis au jour par les recherches en éthologie. D. Guillo rajoute qu'il importe de raisonner de manière réciproque et considérer aussi que les humains apprennent et acquièrent des conduites nouvelles au contact des animaux. C'est donc un mécanisme de «social learning» interspécifique qui explique l'émergence et la diffusion de ces comportements ajustés aux différences des êtres (mais qui n’impliquent pas la résorption de ces différences).

Le chapitre 5 illustre cette idée, en évoquant la manière dont la pratique des chiens guides d'aveugle a émergé depuis le début du $20^{\mathrm{e}}$ siècle, et s'est diffusée grâce à un mécanisme de social learning indirect, puisqu'il n'a jamais impliqué une «copie» des actes du partenaire de l'interaction (le futur chien guide et son éducateur étant bien incapables de reproduire les gestes de l'autre). C'est au contraire un «faire faire» qui se donne à voir dans cette forme de social learning, reposant sur les différences entre humains et chiens, et visant à modeler (accentuer) ces différences dans un sens bien précis. Ainsi, la notion de social learning indirect permet à l'analyste de donner un degré plus élevé de généralité aux interactions situées qu'il observe, en considérant la manière dont elles se diffusent à l'échelle macro-sociale. Prendre pour point de départ l'étude des interactions situées constitue aussi pour D. Guillo la première étape d'une approche résolument «écologique», se focalisant en priorité sur les relations entre les vivants et les manières dont ces relations se déploient de manière réticulaire, synchroniquement et diachroniquement. L'enjeu du sixième chapitre est précisément d'ébaucher la compatibilité possible entre les connaissances actuelles sur 
les mécanismes de sélection naturelle et d'évolution biologique, et des approches centrées sur l'étude des interactions. Là encore, l'auteur place les mécanismes de social learning indirect au centre de l'explication de la transmission et la diffusion des traits comportementaux chez les humains et les animaux. Aux forces évolutives habituellement convoquées pour expliquer ce phénomène (les gènes animaux, les gènes humains et les cultures humaines - via la domestication), D. Guillo propose d'en ajouter une autre : les cultures animales ellesmêmes, ou «les conduites qui se répandent dans les populations animales sous l'effet de leurs interactions avec d'autres espèces, au premier rang desquelles les humains. $»$ (p.231) Les cultures animales sont alors envisagées comme facteurs de transformation des gènes et des conduites socialement acquises d'autres espèces, en particulier les humains. D. Guillo s'appuie ici sur une conception évolutionniste de la domestication envisagée indépendamment de toute référence aux intentions et initiatives humaines. Une telle conception, écrit-il, permet «d'aller plus loin dans la rupture avec l'anthropocentrisme» (p.22) et d'appréhender les cultures animales comme variable à part entière de la coévolution. À ce titre, le nombre croissant d'animaux de compagnie dans les sociétés postindustrielles est décrit par $\mathrm{D}$. Guillo comme un phénomène puissant de coévolution, auquel les animaux eux-mêmes prennent part activement en ajustant par exemple leurs personnalités afin de favoriser leur adoption par des humains. À l'issue de ce chapitre 6, l'interaction - en particulier l'ajustement écologique en situation de différences interspécifiques - est ainsi constituée en point de passage obligé et dénominateur commun d'un programme d'analyse multiniveaux des cultures animales (humaines et non-humaines) appréhendées aux niveaux phylogénétique, ontogénétique et interactionnel.

Le dernier chapitre (chapitre 7) consiste alors à mettre un tel programme à l'épreuve de l'analyse des phénomènes socioculturels proprement humains. Comme dans le cas des études des relations humains-animaux, D. Guillo diagnostique un biais 
identitaire constitutif de l'étude des sociabilités et des cultures humaines depuis la naissance des sciences sociales, au sein desquelles le lien social trouverait sa source dans l'identité partagée, tandis que les différences ne seraient que génératrices de division, de segmentation. Contre un tel biais identitaire, il met l'accent sur la nécessité de prendre en compte la place des différences individuelles dans la construction et la diffusion des sociabilités humaines. Dans les sociétés humaines, les conduites ne se répandent pas sous les seuls effets de l'imitation et de la reproduction : la transmission de certaines compétences s'opère via des ajustements interactionnels profondément asymétriques, dans lesquels l'adoption d'une conduite ou l'acquisition d'une compétence n'est rendue possible que par la différence qui sépare le «récepteur » de l'« émetteur ». L'apprentissage du biberon chez le nouveau-né, ou l'adoption de certains codes vestimentaires chez les fans d'un chanteur célèbre ne s'appuient pas seulement sur l'imitation des parents ou de la vedette. On a affaire, nous dit D. Guillo, à un «faire faire» plutôt qu'à un «faire comme». Développant l'exemple de la socialisation au genre, Guillo défend l'idée d'une diffusion intergénérationnelle des normes qui procéderait d'un social learning indirect : ainsi, l'adoption de conduites supposées propres à un genre serait suscitée par l'interaction avec des individus appartenant à l'autre genre (et se comportant comme tel). Dans cet ultime chapitre, l'auteur rediscute des relations entre certains concepts classiques des sciences sociales et la théorie générale de la culture qu'il cherche à esquisser. Le pouvoir, par exemple, devient un fondement de la culture du «faire faire» car, écrit-il, «le propre du pouvoir est de parvenir à produire chez des êtres - souvent différents - des manières de faire, des attentes et parfois des manières de penser que ceux qui le détiennent n'entendent pas endosser eux-mêmes. $\gg$ (p. 290)

En refermant Les fondements oubliés de la culture, on ne peut qu'être convaincu de l'intérêt du projet de D. Guillo. Dans cet ouvrage ambitieux, l'auteur prend à bras-le-corps des questions fort complexes qu'il parvient à restituer avec beaucoup d'érudition et de pédagogie. L'état de l'art des recherches sur les sociabilités animales dans les sciences du comportement en est une illustration remarquable. On peut tout particulièrement apprécier qu'avec son analyse critique des discussions théoriques et des usages empiriques de la notion d'anthropomorphisme, D. Guillo ne se contente pas de décrypter des débats académiques, parfois confidentiels et laborieux : il y contribue activement, en produi- 
sant des arguments décisifs, extrêmement fins et peu entendus. Grâce à ses analyses, on prend la mesure de l'intérêt pour les sciences sociales de porter le regard sur les relations humains-animaux. La notion de «culture interspécifique» constitue à cet égard une belle réussite, en ce qu'elle permet de bien circonscrire l'objet en l'arrimant à une thématique transversale aux recherches en sciences sociales. La démarche proposée par est également séduisante. D. Guillo Lapproche conceptuelle déflationniste, consistant à approcher ces relations «par le bas», est réellement salutaire, au regard du paysage tuel se dessinant autour de la question animale, 1 ís chargé de généralisations excessives, d'enjeux normatifs, et souvent pauvre empiriquement. Les exemples mobilisés par l'auteur donnent un bon aperçu de la richesse potentielle des terrains encore à explorer. L'arrimage de l'échelle de l'interaction à l'échelle de l'évolution est intéressant, en ce qu'il permet d'aborder la question des changements des rapports humains-animaux, en dépassant une analyse en termes de représentations sociales. Il est également appréciable que $\mathrm{D}$. Guillo mobilise la biologie et les sciences du comportement en récusant tout déterminisme : la nature ne semble pas ici contraignante, et les humains ne sont pas dessaisis de leur capacité d'action, ni de leur responsabilité dans les dynamiques évolutives. Malgré toutes ces vertus, un tel programme suscite un certain nombre de questions de fond, auxquelles nous consacrons la suite de cet article.

\section{Les limites de la purification épistémologiques}

On l'aura compris, le diagnostic de D. Guillo concernant le biais identitaire est essentiellement épistémologique, et c'est à ce titre qu'il parvient à emporter l'adhésion du lecteur : suivant le fil de l'histoire des idées, on comprend très bien pourquoi les «cultures interspécifiques » n'ont pas plus attiré l'attention des chercheurs. Pour autant, à s'en référer uniquement à la littérature produite par la biologie néodarwinienne, l'écologie comportementale, l'étholo- 
gie, les sciences du comportement, et les sciences sociales (la sociologie et l'anthropologie, en particulier), D. Guillo semble négliger d'autres dimensions. Les contextes institutionnels, organisationnels, de la recherche et de l'enseignement supérieur, les asymétries de pouvoir, de financement entre les disciplines, le formatage des publications plus favorables à certains types de paradigmes, etc.: tout ce que la sociologie des sciences a pu nous apprendre à ce sujet est tenu pour quantité négligeable par $\mathrm{D}$. Guillo, qui ne s'engage pas dans une démarche d'enquête lorsqu'il s'agit d'évaluer les travaux qu'il étudie. L'auteur se contente en effet d'indiquer que certains courants étudiés sont «minoritaires», «hétérodoxes», et que d'autres sont «académiques», sans que l'on puisse juger de la validité empirique de cette qualification. Ce qui amène à questionner le traitement réservé par l'auteur aux travaux en sciences sociales abordant les rapports aux animaux, dans son deuxième chapitre. On comprend mal comment ces travaux présentés comme marginaux, tant pour l'éthologie que pour les sciences sociales, pourraient être tenus responsables de l'indifférence réciproque entre ces deux ensembles disciplinaires et de leur difficulté à penser les cultures interspécifiques. Alors même que la lecture de l'ouvrage donne la mesure des réaménagements conséquents que nécessiterait l'application de son programme (une refondation épistémologique), D. Guillo fait peu de cas des difficultés concrètes rencontrées par les recherches en sciences sociales sur les relations anthropozoologiques à trouver une arène académique leur permettant d'aborder un objet paraissant sortir de leur périmètre ${ }^{6}$. Pas plus qu'il ne fournit d'indication sur la forme institutionnelle propice à l'épanouissement du nouveau paradigme qu'il ébauche. Pourtant, D. Guillo aurait pu tirer parti des enseignements riches et abondants de la sociologie du travail scientifique et des institutions disciplinaires ${ }^{7}$ qui montrent que l'histoire des idées ne se résume pas à des arbitrages épistémologiques, mais met en

6. Pour le cas des Animal Studies, voir Jérôme Michalon, «Les Animal Studies peuvent-elles nous aider à penser l'émergence des épistémès réparatrices?», Revue d'Anthropologie des Connaissances, vol.11, №3, 2017, p.321-349. Pour le cas des travaux d'anthropologie «non moderne», inspirés par les STS, on pourrait citer la revue NatureCulture, qui tente de constituer un espace éditorial «hors norme», en adéquation avec l'hybridité des théories qui y sont représentées.

7. Michel Dubois, "Private knowledge" et "programme disciplinaire" en sciences sociales : étude de cas à partir de la correspondance de Robert K. Merton », L'Année sociologique, vol.64, №1, 2014, p.79-119; Timothy Lenoir, Instituting Science: The Cultural Production of Scientific Disciplines, Stanford, Stanford University Press, 1997 ; Séverine Louvel, «Ce que l'interdisciplinarité fait aux disciplines», Revue francaise de sociologie, vol.56, №1, 2015, p.75-103 ; Terry Shinn et Pascal Ragouet, Controverses sur la science. Pour une sociologie transversaliste de l'activité scientifique, Paris, Raisons d'agir, 2005. 
jeu du travail de démarcation ${ }^{8}$ entre des territoires académiques, des approches et des objets de recherche concurrents. Sans renoncer à l'ambition épistémologique, l'intégration de ces dimensions aurait permis de formuler un diagnostic très différent, tout à la fois sur les explications de la prégnance du biais identitaire, et sur les façons d'y remédier. L'analyse qu'il développe sur les difficultés et les réticences des sciences sociales et des sciences de la vie à travailler ensemble, par exemple, aurait pu bénéficier de l'apport des recherches empiriques sur l'interdisciplinarité ${ }^{9}$ qui permettent de penser de façon réaliste les conditions de possibilité des programmes interdisciplinaires. En outre, les travaux empiriques sur les modalités d'articulation entre disciplines ou communautés scientifiques ont déjà fourni un certain nombre de propositions conceptuelles ( «zone d'échanges $»^{10}$, « arène interstitielle $»^{11},\left\langle z\right.$ zone franche $»^{12}$ ) qui auraient permis d'enrichir la proposition d'un «estran» entre sciences sociales et sciences du vivant défendue par D. Guillo.

Cette promotion de l'interdisciplinarité faisant l'économie des apports de la sociologie des sciences n'est certes pas l'apanage de D. Guillo. En effet, il n'est pas le premier à souhaiter articuler les sciences sociales et les sciences de la vie en prenant pour objet privilégié les rapports humains-animaux : en témoignent, entre autres, l'étho-ethnologie de D. Lestel, F. Brunois et F. Gaunet $^{13}$, l'anthropologie de Tim Ingold ${ }^{14}$, l'histoire du point de vue des animaux d'Éric Baratay ${ }^{15}$, ou encore les travaux de Véronique

8. Thomas F. Gieryn, «Boundary-Work and the Demarcation of Science from Non-Science: Strains and Interests in Professional Ideologies of Scientists », American Sociological Review, vol. 48, №6, 1983, p.781-795.

9. Jerry A. Jacobs et Scott Frickel, «Interdisciplinarity: A Critical Assessment», Annual Review of Sociology, vol.35, №1, 2009, p.43-65; Scott Frickel, Mathieu Albert et Barbara Prainsack (eds.), Investigating Interdisciplinary Collaboration: Theory and Practice across Disciplines, New Brunswick (NJ), Rutgers University, 2016 ; Séverine Louvel, « Ce que I'interdisciplinarité fait aux disciplines», art. cit. ; Dominique Vinck, Pratiques de l'interdisciplinarité : mutations des sciences, de l'industrie et de l'enseignement, Grenoble, Presses universitaires de Grenoble, 2000.

10. Peter Galison, Image \& Logic: A Material Culture of Microphysics, Chicago, The University of Chicago Press, 1997.

11. Bernward Joerges et Terry Shinn, Instrumentation Between Science, State and Industry, Berlin, Springer Science \& Business Media, 2001.

12. Anne Marcovich et Terry Shinn, «Where is disciplinarity going? Meeting on the borderland», Social Science Information, vol.50, №3-4, 2011, p.582-606.

13. Dominique Lestel, Florence Brunois et Florence Gaunet, «Etho-ethnology and ethno-ethology», Social Science Information, vol.45, №2, 2006, p.155-177.

14. Tim Ingold (eds.), What is an animal?, Londres \& New York, Routledge, 1987.

15. Éric Baratay, «Les socio-anthropologues et les animaux. Réflexions d'un historien pour un rapprochement des sciences », Sociétés, №108, 2010, p.9-18 ; Éric Baratay, Le point de vue animal. Une autre version de l'histoire. Paris, Seuil 2012. 
Servais $^{16}$, Florent Kohler ${ }^{17}$, Nicolas Lescureux ${ }^{18}$, Vincent Leblan ${ }^{19}$. Aucun de ces auteurs n'a appelé à une collaboration entre les disciplines autrement qu'en des termes épistémologiques, négligeant ses tenants et aboutissants institutionnels. De ce point de vue, le programme de D. Guillo n'est pas plus novateur que celui de ses prédécesseurs ou de ses contemporains. Par ailleurs, si certains de ces travaux sont mentionnés par l'auteur, la plus-value de son programme par rapport aux autres n'est jamais réellement discutée dans l'ouvrage. Ce problème de positionnement se retrouve également lorsque D. Guillo passe en revue les travaux en sciences sociales sur les relations humains-animaux. Le critère de regroupement choisi est éminemment discutable: tous ces travaux ne se revendiquent pas de la remise en cause du Grand Partage, tous ne cherchent pas à rendre compte de l'agentivité animale, et tous, enfin, ne cultivent pas une méfiance vis-à-vis des sciences de la nature. En fait, il serait plus honnête de reconnaître à cet ensemble de recherches une certaine indétermination quant à ses objets et ses objectifs : une hésitation entre la volonté de connaître les relations humains-animaux et celle de connaître les animaux, deux démarches assez différentes, qui n'engagent pas les mêmes enjeux épistémologiques et méthodologiques ${ }^{20}$. Cette indétermination relative devrait inciter à la prudence qui voudrait se livrer à une critique purement épistémologique. D’autant que cette oscillation se retrouve chez D. Guillo, qui présente son programme comme le plus à même de rendre compte des relations humains-animaux et de l'agentivité de ces derniers, comme s'il existait une continuité logique entre ces deux objectifs. En fait, les raisons de cette association pourraient aussi être «socio-logiques » : la montée en puissance inédite de revendications morales sur la condition animale ${ }^{21}$

\footnotetext{
16. Véronique Servais, «Faut-il faire la sociologie des singes?», SociologieS [en ligne], journals.openedition.org/sociologies/4054, 2012 ; Véronique Servais, La science [humaine] deschiens, Lormont, Le bord de l'eau, 2016.

17. Florent Kohler, «Sociabilités animales. Introduction», Études rurales, №189, 2012, p.11-31.

18. Nicolas Lescureux, «Towards the necessity of a new interactive approach integrating ethnology, ecology and ethology in the study of the relationship between Kirghiz stockbreeders and wolves », Social Science Information, vol.45, №2, 2006, p.463-478.

19. Vincent Leblan, Aux frontières du singe. Relations entre hommes et chimpanzés au Kakandé, Guinée (19e-21e siècle), Paris, Éditions de l'EHESS, 2017.

20. Jérôme Michalon, Antoine Doré et Chloé Mondémé, «Une sociologie avec les animaux: faut-il changer de sociologie pour étudier les relations humains/animaux?», SociologieS [En ligne], Dossier «Sociétés en mouvement, sociologie en changement», mis en ligne le 7 mars 2016, sociologies.revues.org/5329.

21. Fabien Carrié et Christophe Traïni (dir.), S'engager pour les animaux, Paris, Presses universitaires de France, 2019. Christophe Traïni, La cause animale (1820-1980). Essai de sociologie historique, Paris, Presses universitaires de France, 2011.
} 
n'est sans doute pas pour rien dans l'apparition de ce double impératif scientifique, consistant à connaître les relations aux animaux et à rendre justice à leur capacité d'action ou à leur point de vue. Ce lien est par exemple très clair pour les recherches étiquetées «animal studies», qui affirment leur proximité avec le militantisme pro-animaux et son influence sur leur épistémologie ${ }^{22}$. Là encore, la primauté accordée par D. Guillo à l'épistémologie pour rendre compte des apories de certaines recherches - et par-delà, des raisons du biais identitaire - ne convainc que partiellement.

\section{Moderniser Latour?}

Outre l'inscription dans un «air du temps», qui invite à relativiser la rupture paradigmatique annoncée, on trouve au programme de $\mathrm{D}$. Guillo un «air de famille» avec une ouvre pourtant copieusement attaquée dans l'ouvrage : celle de Bruno Latour. Au début des années 1980, Bruno Latour et la primatologue Shirley Strum ont entamé une col- - laboration visant à examiner les manières dont chacune de leur discipline (sociologie et éthologie) définissait théoriquement le «social», et à quelle échelle elle le documentait empiriquement ${ }^{23}$. Plus de 30 ans avant D. Guillo, S. Strum et B. Latour écrivent que c'est par l'étude des interactions (approche commune aux deux disciplines, et, à l'époque, innovante) que l'on pourra réellement comprendre les dynamiques sociales, à la fois chez les humains et chez les animaux ${ }^{24}$. Les réflexions de B. Latour et S. Strum ne s'appliquaient certes pas aux rela-

22. Jérôme Michalon, «Les Animal Studies peuvent-elles nous aider à penser l'émergence des épistémès réparatrices?», art. cit. Jérôme Michalon, «La cause animale et les sciences sociales : influences, dévoilements et appariements», in Fabien Carrié et Christophe Traïni (dir.), S'engager pour les animaux, op.cit., p. 89-101.

23. Bruno Latour et Shirley Strum, «Human social origins: Oh please, tell us another story», Journal of Social and Biological Structures, vol.9, №2, 1986, p.169-187. Pour une analyse détaillée de cette collaboration, voir Nicolas Langlitz, «Primatology of Science: On the Birth of Actor-Network Theory from Baboon Field Observations », Theory, Culture \& Society, vol.36, №1, 2019, p.83-105.

24. Shirley Strum et Bruno Latour, «Redifining the social link: from baboons to humans», Social Science Information, vol.26, №4, 1987, p.783-802. 
tions interspécifiques, et on pourrait leur reprocher d'avoir réintroduit une frontière entre sociétés humaines et sociétés animales ${ }^{25}$.

Pour autant, une autre collaboration, celle - plus connue - que B. Latour a nouée avec Michel Callon, a fourni l'outillage conceptuel propre à dépasser ces objections. Quand D. Guillo propose de s'intéresser aux interactions entre humains et animaux, avec le minimum de conceptualisations préalables sur les propriétés des entités en présence, pour pouvoir mieux comprendre ce que produisent ces interactions (de l'identité ou de la différence), n'est-ce pas en substance la définition du «principe de symétrie généralisée » de Latour et Callon ${ }^{26}$ ? La symétrie généralisée est un plaidoyer pour l'étude d'interactions situées, où les différences entre savoirs profanes et savoirs experts, nature et culture, entités humaines et non humaines, ne seraient pas tenues pour acquises préalablement à l'observation. Cet agnosticisme méthodologique a souvent été mal compris, voire caricaturé : niant toute différence entre les êtres, il entretiendrait une confusion ontologique. Mais en réalité, B. Latour et M. Callon expliquent que, si le principe de symétrie implique bien cette mise à plat ontologique a priori, ce n'est que pour mieux voir, a posteriori, comment les différences (ou les identités) entre les êtres se construisent et s'actualisent dans l'interaction. Il ne s'agit pas de nier les différences ontologiques, mais d'objectiver à nouveau frais leur constitution.

On retrouve exactement ces aspects (déflation ontologique préalable à l'observation des interactions, permettant de comprendre la formation des différences) chez D. Guillo, qui les présentent malgré tout comme des innovations. Tout comme Latour $^{27}$, D. Guillo va puiser dans l'interactionnisme les ressour-

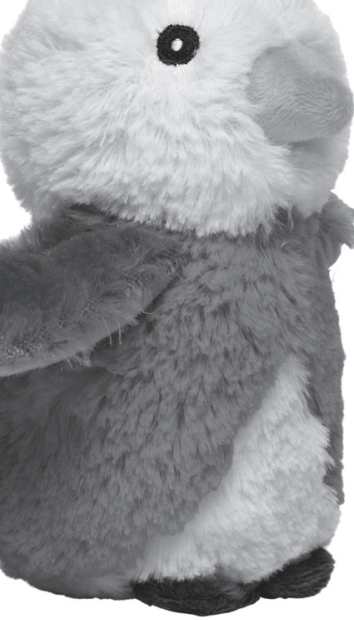

25. Parl'introduction de la différence entre des sociétés complexes, chez les babouins, et des sociétés compliquées, chez les humains. Voir Antoine Doré et Jérôme Michalon, «What makes human-animal relations "organizational"? The De-Scription of anthrozootechnical agencements », Organization, vol.24, №6, 2016, p.761-780.

26. Madeleine Akrich, Michel, Callon et Bruno Latour, Sociologie de la traduction. Textes fondateurs. Paris, Presses des Mines, 2006 ; Bruno Latour, «Le Grand Partage», Revue du MAUSS, №1, 1988, p.27-65 ; Bruno Latour, Nous n'avons jamais été modernes. Essai d'anthropologie symétrique, Paris, La Découverte, 1991.

27. Bruno Latour, «Une sociologie sans objet? Note théorique sur l'interobjectivité », Sociologie du travail, vol.36, №4, 1994, p.587-606. 
ces intellectuelles nécessaires pour penser comment humains et non humains peuvent «faire société ». Tout comme Latour ${ }^{28}$, D. Guillo en vient à défendre une conception performative de la société, refusant de considérer l'existence de structures sociales surplombantes. Tout comme Latour ${ }^{29}$, D. Guillo explique que c'est dans les réseaux que les rapports de pouvoir, renégociés au cours des interactions, se distribuent et se cristallisent. Tout comme Latour, D. Guillo pense que c'est seulement en suivant ces réseaux que l'on arrivera à dépasser le grand dualisme nature/culture et les frontières disciplinaires. Tout comme Latour ${ }^{30}$, D. Guillo plaide pour une approche «écologique», se focalisant sur les relations plutôt que sur les entités. Tout comme Latour ${ }^{31}$, D. Guillo place le «faire faire» au centre de l'explication des dynamiques écologiques (et non plus seulement «sociales »). Mais sa définition du «faire faire», nous dit-il, n’a rien à voir avec celle de Latour. À la lecture de l'ouvrage, ce n'est pas si clair, et on en vient à penser que la notion de social learning indirect sert pratiquement de synonyme au «faire faire» latourien ; voire de traduction. Si les travaux de B. Latour n'ont pas été appropriés par les éthologues et les biologistes, c'est sans doute qu'il leur manquait un traducteur, les rendant compatibles avec l'épistémologie «moderne» dans laquelle ces sciences s'ancrent. L'entreprise de D. Guillo aura-t-elle finalement pour effet inattendu de moderniser Latour, en le rendant audible par les sciences de la vie?

\section{Sans technique, ni institutions: où se solídifie la culture?}

Convoquant la collaboration Latour/Strum, on voit que l'ambition d'articuler sciences de la nature et sciences sociales en privilégiant l'interaction n'est pas nouvelle ${ }^{32}$. De la même façon, re-

28. Ibid. Et aussi : Shirley Strum et Bruno Latour, «Redifining the social link», art. cit.

29. Bruno Latour, Nous n'avons jamais été modernes, op. cit.

30. Bruno Latour, Cécile Schwartz et Florian Charvolin, «Crises des environnements, défis aux sciences humaines», Futur Antérieur, №6, 1991, p. 28-56; Bruno Latour, «Moderniser ou écologiser. À la recherche de la Septième Cité», Écologie \& politique, №13, 1995, p.5-27.

31. Bruno Latour, Petite réflexion sur le culte moderne des dieux faitiches, Paris, Les Empêcheurs de penser en rond, 1996 ; Bruno Latour, «Facture/Fracture. De la notion de réseau à celle d'attachement », in André Micoud et Michel Peroni (dir.), Ce qui nous relie, La Tour d'Aigues, Éditions de L'Aube, 2000, p.189-208.

32. Au point que N. Langlitz propose d'interpréter la théorie de l'acteur-réseau comme un prolongement de l'anthropologie évolutionnaire. Voir Nicolas Langlitz, «Primatology of Science: On the Birth of Actor-Network Theory from Baboon Field Observations », Theory, Culture \& Society, vol.36, №1, p.83-105. 
penser la notion de «culture » en s'appuyant sur les recherches en éthologie a connu un précédent, étonnamment absent de l'ouvrage. En 2001, paraissait en effet un livre qui débutait ainsi : «Dans les pages qui suivent, je soutiens la thèse selon laquelle loin de s'opposer à la nature, la culture est un phénomène qui est intrinsèque au vivant dont elle constitue une niche particulière, qu'on en trouve les prémices dès les débuts de la vie animale [...].» ${ }^{33}$ Dans Les origines animales de la culture, dont le seul titre aurait justifié que D. Guillo lui consacre ne serait-ce que quelques lignes, Dominique Lestel prenait acte du développement des travaux en éthologie sur les «cultures animales ». Celles-ci, écrivait-il, nous imposent «de devoir penser le phénomène culturel dans une perspective évolutionniste ${ }^{34}$. En cohérence avec cette proposition, D. Lestel (avec ses collègues Florence Brunois et Florence Gaunet) appellera quelques années plus tard au développement d'une «nouvelle science», synthèse « écologique» entre éthologie et ethnologie ${ }^{35}$. Malgré la parenté entre les projets de D. Lestel et de D. Guillo, rien n'est dit du premier dans l'ouvrage du second.

La référence à $\mathrm{D}$. Lestel aurait sans doute permis à $\mathrm{D}$. Guillo de se positionner plus explicitement par rapport à tout un pan des recherches dans lesquelles la culture a été pensée dans ses rapports avec les «non humains ». Nous pensons en particulier à l'anthropologie des techniques, qui s'est développée en France sous l'impulsion d'André Leroi-Gourhan ${ }^{36}$, puis à travers les recherches sur la domestication de André-Georges Haudricourt ${ }^{37}$, et dans les pages de Techniques et Cultures. Cette revue s'est ouverte dès les années 1990 aux travaux portant sur l'utilisation et la fabrication d'outils chez certains primates (les chimpanzés notamment) - une «découverte» qui a eu un écho retentissant dans cette communauté qui pendant longtemps avait tenu la technique comme critère de différenciation entre humains et animaux. Ce qui ouvrira la voie à une anthropologie des techniques des animaux $^{38}$ accompagnant l'affirmation par les éthologues de l'existence de cultures animales. C'est dans cette filiation qu'il faut

\footnotetext{
33. Dominique Lestel, Les origines animales de la culture, Paris, Flammarion, 2001, p. 8.

34. Ibid., p.8.

35. Dominique Lestel, «Ethology and ethnology: the coming synthesis. A general introduction », Social Science Information, vol.45, №2, 2006, p.147-153; Dominique Lestel, Florence Brunois et Florence Gaunet, «Etho-ethnology and ethno-ethology», art.cit.

36. André Leroi-Gourhan, Milieu et techniques, Paris, Albin Michel, 1945.

37. André Georges Haudricourt, «Domestication des animaux, culture des plantes et traitement d'autrui», L'Homme, vol.2, №1, 1962, p.40-50.

38. Frédéric Joulian, «Techniques du corps et traditions chimpanzières », Terrain, №34, 2000, p.37-54.
} 
réinscrire l'ouvrage de D. Lestel : pour lui, l'utilisation d'objets techniques par les animaux remet en cause une définition de la culture réservée à l'espèce humaine. Il serait dès lors réducteur de ne présenter la contribution de l'anthropologie des techniques française qu'à travers les analyses qu'elle a produites sur la domestication. La critique d'anthropocentrisme adressée par D. Guillo à ces analyses ${ }^{39}$ ne vaut en effet que pour une partie d'entre elles, qui limitent le rôle des animaux dans la production de la culture à celui de réceptacle passif des pratiques humaines. Avec une autre partie de ces travaux, dans lesquels la technique n'est plus l'apanage des humains, la voie est ouverte pour repenser en profondeur la notion de culture, et en produire une version dans laquelle l'agentivité des animaux serait prise en considération ${ }^{40}$. Reconnaissons néanmoins que cette voie n'a pas été explorée dans les proportions souhaitées par D. Guillo et que l'étude des «cultures interspécifiques » reste encore à consolider.

Évoquer Techniques \& Cultures amène à aborder l'une des caractéristiques de l'ouvrage de D. Guillo, consistant précisément à penser la culture sans technique. Pour l'auteur, nous l'avons dit, la culture prend la forme d'une suite d'ajustements interactionnels, au sein desquels les différences entre les êtres s'expriment et se construisent. Ces ajustements interactionnels se multiplient de manière réticulaire, sous l'effet, non pas de l'imitation, mais du «faire faire». Mais une question demeure sous-explorée : comment ces patterns interactionnels se stabilisent-ils et durent-ils dans le temps? Quelles forces cana- lisatrices cadrent et limitent

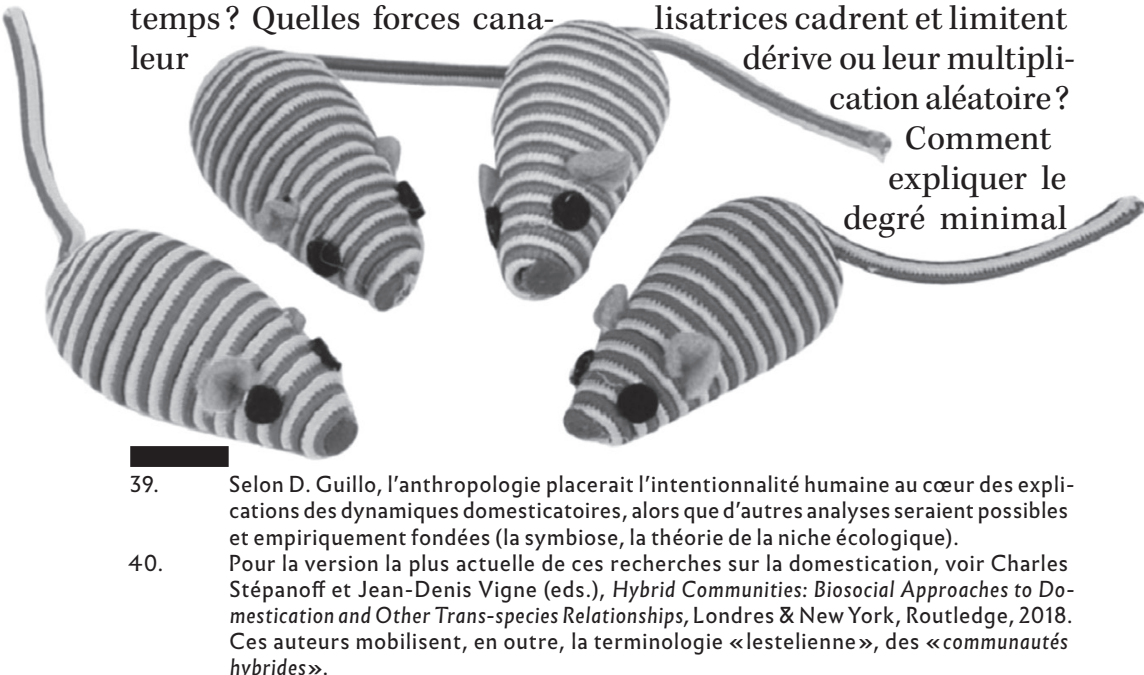
hybrides». 
d'unité et de régularité des motifs interactionnels qui se déploient en deçà et par-delà la frontière des espèces, et qui constituent ce que D. Guillo semble définir comme une culture? En bref, à quoi tient cet ordre social particulier appelé culture? Pour répondre à cette question, revenons un instant sur la collaboration entre B. Latour et S. Strum. Dans leur article de 1986 (antérieur, donc, aux travaux sur les cultures animales), ils proposent aussi de faire de l'utilisation des objets techniques un critère de différenciation anthropozoologiques : les sociétés des baboins et celles des humains seraient différentes, car les premières n'utiliseraient pas d'objets techniques, et seraient par conséquent condamnées à renégocier sans cesse leur ordre social. Au contraire, nous disent S. Strum et B. Latour, les sociétés humaines ne se seraient jamais construites sans les objets techniques qui assurent la reproduction de l'ordre social en le «solidifiant» et en le pérennisant par-delà les générations. Grâce aux objets techniques, une société est autre chose qu'une succession d'interactions «nues », vouées à être répétées indéfiniment par ses membres, ne pouvant compter que sur leurs ca-

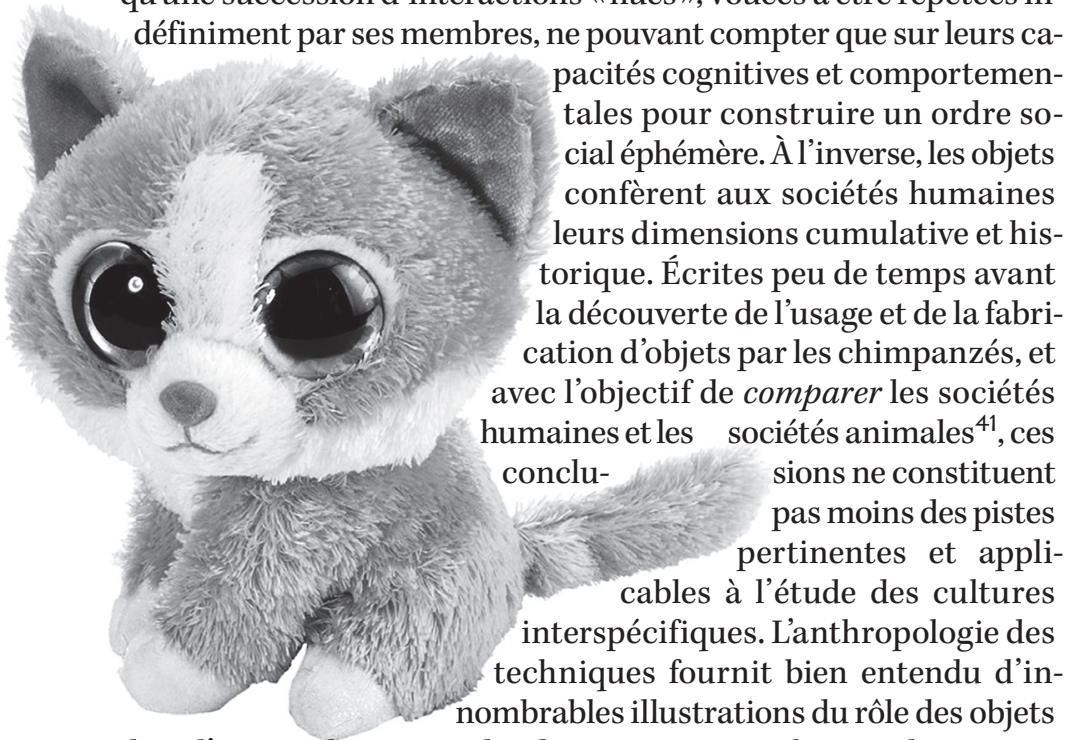
dans l'accomplissement des dynamiques sociales interhumaines, et leur reproduction dans l'espace et le temps (d'où sa proximité avec l'anthropologie culturelle, ou même l'archéologie, pour laquelle les objets, leur analyse, leur recueil, leur conservation, ne sont pas la moindre des préoccupations).

41. Elles sont, à ce titre, tributaires du biais identitaire critiqué par D. Guillo. 
À l'aune de ces recherches, une description des sociétés et a fortiori des cultures - humaines dépourvues d'objets apparaît bien peu réaliste. Il en va de même pour les interactions humains-animaux, et l'on s'en rend compte à la lecture de plusieurs situations analysées par D. Guillo. Par exemple, c'est bien autour de l'ouverture d'une baie vitrée que se joue l'interaction entre l'auteur (on présume) et son chien. Que ces objets puissent «cadrer » les interactions interspécifiques, leur donner une forme particulière, ne semble pas retenir l'attention de D. Guillo; pas plus que le fait que ces objets aient été fabriqués par des humains, et que les animaux les utilisent ou s'y adaptent. En un mot : le pouvoir cadrant, voire contraignant, des objets humains sur les interactions interspécifiques n'est pas pris en compte pour expliquer la multiplication des conduites réglées entre humains et animaux. Or, il $\mathrm{y}$ aurait tout à fait lieu de penser que les objets techniques ont un rôle important à jouer dans le « faire faire» entre humains et animaux, non seulement parce qu'ils reproduisent et démultiplient la capacité d'action des humains, mais également parce qu'ils permettent de mettre en lumière la part d'inventivité, de résistance, d'agentivité que les animaux mobilisent pour entrer en relation avec nous ${ }^{42}$. Pour cette raison, et aussi parce qu'ils constituent l'ordinaire des interactions humains-animaux ${ }^{43}$, la prise en compte des «agencements anthropozootechniques ${ }^{44}$ pourrait tout à fait s’articuler avec le projet d'objectiver les «cultures interspécifiques».

Passer par l'interaction «nue» pour aborder les cultures interspécifiques n'est donc pas une fatalité. Pas plus que ne l'est l'abandon de toute référence aux entités habituellement convoquées par les recherches sur la culture, et plus largement par les sciences sociales : marchés, gouvernements, lois, religions, etc. En un mot: les institutions. On peut reconnaître le caractère quelque peu éthéré de ces entités, souvent difficilement objectivables en elles-mêmes (à moins, là encore, de les aborder à travers leur matérialité). Il n'en reste pas moins qu'elles ont une influence certaine sur les interactions et sur leur diffusion. L'exemple des chiens

42. Pour un exemple simple, celui de la laisse dans les interactions humains-chiens, voir Antoine Doré et Jérôme Michalon, «What makes human-animal relations “organizational"?», art.cit.

43. Sous l'influence de l'inexorables anthropisation des milieux, il devient de plus en plus difficile d'appréhender les interactions intra et interspécifiques entre d'innombrables animaux non humains sans s'interroger sur les effets de cadrages - intentionnels ou collatéraux - produits par les artefacts techniques humains. D. Guillo en fait mention, sans pour autant y accorder un développement conséquent.

44. Antoine Doré et Jérôme Michalon, «What makes human-animal relations “organizational», art.cit. 
guides d'aveugles, pris par D. Guillo, est à cet égard très parlant : sans la création des associations et des écoles de chiens guides, la rédaction de méthodes d'éducation, de sélection génétique, voire la standardisation ${ }^{45}$, toutes les compétences développées par ces chiens au contact de leurs éducateurs (et réciproquement) n’auraient pas pu se diffuser dans l'espace et dans le temps. Si elles ne cadrent sans doute pas intégralement les interactions situées, les institutions constituent un catalyseur non négligeable lorsqu'il s'agit de faire se multiplier des conduites réglées.

Ainsi, dépouillée des artefacts techniques et institutionnels, la vision de la culture proposée par D. Guillo se révèle parfois moins éclairante que des approches plus classiques. Pour reprendre un autre exemple développé dans l'ouvrage, comment comprendre l'activité coopérative entre des chiens de recherche au sang et des chasseurs sans considérer les modalités d'usage de la laisse, du sifflet, les techniques de sélection des chiens qui conviennent à cette chasse, les institutions qui encadrent et rendent possible cette sélection, mais aussi celles qui définissent le cadre juridique au sein duquel peut s'exercer cette pratique, etc.? Cette coopération entre des chiens de recherche au sang et des chasseurs produit une compétence interspécifique qui n'est possible que parce qu'elle est médiée et équipée de manière directe et indirecte par des artefacts techniques et institutionnels. La réussite d'une recherche au sang repose sur une compétence largement distribuée entre des animaux, des humains et des objets techniques en situation et cadrée par d'autres animaux, humains et objets qui ne sont pas directement partie prenante de la situation de chasse, mais qui la rendent possible ou qui la contraignent.

Renonçant aux objets et aux institutions, D. Guillo se coupe d'une partie des ressources qui lui permettraient de définir ce qui stabilise les patterns interactionnels et d'expliquer leur histoire. D’une partie seulement : n'oublions pas que la réflexion de l'auteur se veut en effet une contribution aux discussions sur les rapports gènes/cultures dans une perspective évolutionnaire. L'ouvrage décrit les cultures animales, au sens d'ensemble de comportements appris par social learning indirect, comme des forces évolutives (p.232), s'ajoutant aux classiques vecteurs génétiques, ontogénétiques et phylogénétiques. L'interaction se retrouve ainsi bien prise 
dans une dynamique historique, au cour d'une dialectique de la coévolution, définie comme le produit de l'action des gènes sur les cultures et, réciproquement, de l'action des cultures sur les gènes. Mais comme (1) la culture selon D. Guillo semble être essentiellement performative (« la culture se fait»), se donnant à voir dans les actions croisées des humains et des animaux, et comme (2) l'auteur refuse d'aborder la culture au travers des artefacts techniques et institutionnels, on peut se demander où est conservée la trace des interactions passées, et ce qui conditionne les interactions futures; bref : où se sédimente la culture? À défaut de mention explicite de toute autre entité, on croit discerner que c'est bien du côté des gènes qu'il faut aller chercher la réponse de D. Guillo.

Cette interprétation trouve des appuis dans l'adhésion patente de D. Guillo à l'épistémologie des sciences de la nature : la seule définition de la culture utilisée et discutée dans l'ouvrage provient de ces sciences. Le concept clé de la proposition de Guillo (le social learning) leur est emprunté, ainsi que l'appel récurrent à la «parcimonie», notion faisant consensus dans les behavioral sciences et en biologie de l'évolution, mais peu mobilisée en sciences sociales. Par ailleurs, c'est bien plus aux sciences sociales qu'aux behavioral sciences que l'auteur fait porter la responsabilité d'une impossible synthèse autour des cultures interspécifiques. Contrairement à ce qu'elles affirment, les sciences sociales seraient les plus promptes à entériner le dualisme nature/culture et les frontières entre les disciplines, par «idéologie» ou «corporatisme». À l'inverse, la sociobiologie, l'écologie comportementale ou la psychologie évolutionniste, en considérant l'humain comme un vivant parmi d'autres, proposent d'abolir ces frontières et ces dualismes, et apparaissent presque,

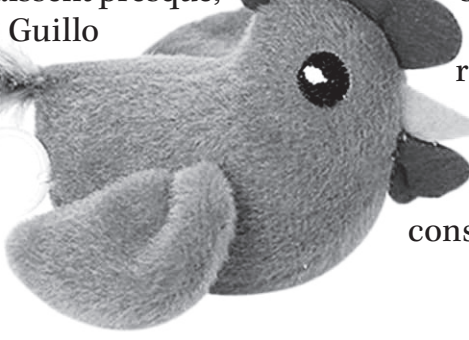
sous la plume de comme des théories progressistes, là où les sciences sociales ne brilleraient que par leur conservatisme. 


\section{Le biais différentialiste : peut-on et doit-on dissocier identité et différence?}

L'identification du biais identitaire, comme démarche analytique postulant et accentuant la similitude des êtres, est sans conteste un apport fondamental de l'ouvrage. Mais à trop forcer le trait, D. Guillo tomberait presque dans les travers qu'il dénonce. En effet, à lire l'ouvrage, on a l'impression qu'il existe un rapport pratiquement causal entre la caractérisation produite par l'observateur (le postulat ontologique), affirmée en amont de l'observation («intéressons-nous à ces interactions, en considérant que les êtres qui y sont engagés sont différents/similaires ») et la caractérisation des êtres, une fois l'interaction observée («ayant interagi, nous constatons que la différence/similitude entre les êtres s'est accentuée ou résorbée»); comme si, finalement, sous le regard de l'analyste, l'identité postulée en input ne pouvait produire que de l'identité constatée en output. Le programme proposé par l'auteur paraît consister en un renversement de cette mécanique : pour se déprendre du biais identitaire, dit D. Guillo, il faut s'intéresser à des situations où les êtres sont différents (ce qui est un postulat ontologique), pour pouvoir observer ce que produisent leurs interactions en termes de renforcement de ces différences ${ }^{46}$. La différence postulée en input implique nécessairement de la différence observée en output. Comment ne pas voir dans ce renversement, la rigidification d'une relation causale entre input et output? Se substituant au biais identitaire, un biais différentialiste apparaît nettement dans le programme proposé par l'auteur. Une fois ce biais identifié, il devient possible d'en évaluer les effets en termes de gain et de coût cognitif.

Revenons ainsi sur la proposition d'application du programme de D. Guillo aux objets bien identifiés en sciences sociales. Constatons d'abord qu'en cherchant à réhabiliter l'étude

46. On note ici plusieurs oppositions entre le programme de D. Guillo et celui de B. Latour et $M$. Callon : en input, le principe de symétrie propose en effet une forme de déflation ontologique pour aborder les interactions entre des êtres sans préjuger a priori, ni de leur différence, ni de leur similitude. D. Guillo, lui, propose de postuler que les êtres sont différents, mais que ces différences ne sont pas des obstacles insurmontables pour l'observateur - voire même qu'elles sont épistémologiquement fertiles. Pour autant, comme Latour et Callon, Guillo recommande, à des fins méthodologiques, de ne pas leur donner trop d'importance. En outre, le principe de symétrie laisse ouverte la possibilité que leurs interactions puissent produire, en output, de la différence ou de la similitude entre les êtres (ou les deux à la fois). Le programme de D. Guillo semble au contraire ne pas intégrer cette potentialité. 
des différences contre la focalisation sur les identités, D. Guillo en arrive à formuler un diagnostic parfois sévère, voire partial, sur les sciences sociales qui, écrit-il, auraient très largement négligé la prise en compte des différences dans l'étude des mondes sociaux. Pour les sociologues, les différences seraient problématiques, et il s'agirait de les résorber (au prisme d'une interprétation marxisante) ou de faire en sorte qu'elles ne se multiplient pas trop (dans une conception plus durkheimienne), sans quoi elles menaceraient l'existence même d'un lien social. Le souci de Durkheim pour le maintien des solidarités face à l'accroissement de la différenciation des organes sociaux est ainsi interprété par D. Guillo comme l'expression d'une panique morale antidifférentialiste : pour Durkheim, la différenciation serait un problème, qui à défaut d'être abolie, devrait être $a$ minima régulée, faute de quoi la société n'existerait plus. Évoquant les recherches portant sur les classes sociales, D. Guillo explique, par ailleurs, que leur trop grande attention à la formation d'identités de classe les a conduits à considérer les différences comme les signes d'une absence de relation sociale, et à négliger le potentiel relationnel des différences produites entre les groupes sociaux. Une telle interprétation semble faire abstraction du fait que les recherches sur les classes sociales n'existent le plus souvent que pour penser les inégalités sociales (soit les différences entre les groupes sociaux). De plus, l'imprégnation marxiste d'une bonne partie de ces recherches laisse à penser que la différence n'y est en aucun cas assimilée à l'absence de lien social, mais bien au contraire à l'existence d'un lien social conflictuel, dont on ne peut pas faire l'économie si l'on veut par exemple comprendre les cultures ouvrières. Dans la même lignée, l'analyse de D. Guillo sur la socialisation aux rôles de genre interroge. Avoir recours à la notion de social learning indirect («croisé » cette fois), permettrait en effet selon l'auteur de comprendre que les rôles différenciés de genre ne se construisent pas uniquement dans l'interaction avec des personnes du même genre (par imitation donc), mais aussi dans les interactions répétées avec des personnes de l'autre genre (par «faire faire $»)$ : preuve supplémentaire que l'accentuation des différences provient du contact avec des entités différentes. La lecture des analyses interactionnistes du genre, fleurissantes depuis la publication de l'Arrangement des Sexes d'E. Goffman ${ }^{47}$, aurait 
sans doute évité à $\mathrm{D}$. Guillo de présenter comme une découverte le fait que l'on devient femme au contact des hommes, et réciproquement. Cela lui aurait également évité de faire de cet exemple du genre l'ultime illustration de l'intérêt d'abandonner le biais identitaire au profit du biais différentialiste : car, comme dans le cas des classes sociales, l'identité d'un genre se renforce en même temps que les différences entre les genres s'affirment.

En fait, un grand nombre de recherches en sciences sociales, des plus classiques aux plus récentes, prennent pour acquis l'intérêt de considérer la production simultanée des similitudes et des différences. Pour l'anthropologie, par exemple, la culture est vue depuis longtemps comme le résultat d'activités sociales produisant conjointement l'identité d'un groupe et son altérité ${ }^{48}$. La création d'identité est ainsi pensée comme consubstantielle de la production de différences. Remplacer le biais identitaire par le biais différentialiste reviendrait donc à dissocier artificiellement ce couple, et à entériner l'impossibilité de penser, dans un même mouvement, production d'identités et production de différences.

Il faut, en outre, introduire une nuance supplémentaire. Les approches «classiques» en sciences sociales cherchent à documenter non seulement la production simultanée des identités et des différences «objectives», mais aussi et surtout, la manière dont ces identités et ces différences sont thématisées par les humains comme significatives (D. Guillo parlerait d'identité ou de différence «subjectives »). Tout le programme de D. Guillo - ou presque - est consacré aux premières, et la volonté de tenir ensemble sciences de la vie et sciences sociales par le truchement d'un interactionnisme radical, épuré de tout «mentalisme» (ennemi commun de l'interactionnisme et de l'éthologie), semble impliquer de rompre avec une quantité considérable de travaux en sciences sociales. À la lecture de l'ouvrage, on comprend bien les raisons qui poussent $\mathrm{D}$. Guillo à vouloir se détacher d'une telle posture, jugée coupable de biais identitaire (favorisant l'empathie et reposant sur elle, donc sur l'identité partagée, selon l'auteur) et suspectée d'anthropocentrisme (car ayant souvent recours à des méthodes d'enquête «logocentrées »). Mais on s'interroge égale-

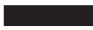

Gender"», Gender and Society, vol.1, №2, 1987, p.125-151; ou Candace West et Sarah Fenstermaker, «Doing Difference», Gender and Society, vol. 9, №1, 1995, p. 8-37, auxquels la revue Terrains et Travaux a consacré un numéro spécial en 2006 : cairn-info. inshs.bib.cnrs.fr/revue-terrains-et-travaux-2006-1.htm.

48. Comme le montrent les travaux de Fredrick Barth (ed.), Ethnic Groups and Boundaries: The social organization of culture difference, Londres, George Allen \& Uwin, 1969.
} 
ment sur le coût cognitif d'une telle proposition : la focalisation sur les comportements ajustés, prônée par D. Guillo, au nom du rejet de l'anthropocentrisme, amènerait-elle à ne plus s'intéresser du tout à ce rapport symbolique? Pour étudier les cultures interspécifiques, faudrait-il renoncer au potentiel heuristique largement éprouvé des formes d'enquêtes plus logocentrées, qui caractérisent souvent les approches structuralistes, communicationnelles, ou encore compréhensives? Le rejet de l'anthropocentrisme nécessite-t-il que l'on se prive des enseignements tirés des travaux sur la construction des rapports symboliques de l'individu au groupe de semblables et à l'altérité?, etc. Se focalisant sur une interaction «nue», dépouillée comme nous l'avons vu d'une partie importante de sa consistance, D. Guillo pense avoir trouvé l'antidote le plus efficace à toute forme de mentalisme. Mais le remède suffit-il à faire disparaître les phénomènes mentaux et leur influence potentielle sur les interactions?

Outre l'étude des différences et des identités «objectives», il n'est pas inutile lorsque l'on s'intéresse aux interactions, d'appréhender les différences et les identités perçues, ressenties, conceptualisées, réfléchies et discutées par les individus. Car la perception est la matrice de l'interaction ${ }^{49}$, et ce n'est généralement que si les identités ou les différences sont perçues par les individus en présence qu'elles peuvent devenir des appuis ${ }^{50}$, ou des prises ${ }^{51}$, dans l'ajustement plus ou moins réciproque des conduites. Une bonne part de la sociologie pragmatique s'est ainsi réapproprié l'héritage de l'interactionnisme symbolique, pour étudier l'influence réciproque des pratiques de catégorisations sur les interactions. Certains auteurs de ces travaux ont eu à cour d'aborder les relations humains-animaux dans cette perspective $\mathrm{e}^{52}$. On trouve également une brillante illustration de cet intérêt croisé pour la question des différences et des identités objectives et subjectives, appliquée aux rapports aux animaux dans les recherches de Vinciane Despret ${ }^{53}$.

49. James Gibson, The ecological approach to visual perception, Hillsdale (NJ), Lawrence Erlbaum Associates, Inc, 1979.

50. Nicolas Dodier, «Les appuis conventionnels de l'action. Éléments de pragmatique sociologique», Réseaux, vol.11, №62,1993, p.63-85.

51. Christian Bessy et Francis Chateauraynaud, Experts et Faussaires. Pour une sociologie de la perception, Paris, Métailié, 1995.

52. Catherine Rémy, La fin des bêtes. Une ethnographie de la mise à mort des animaux, Paris, Economica 2009. ; Catherine Rémy et Myriam Winance, «Pour une sociologie des "frontières d'humanité" », Politix, vol.23, №90, 2010, p.9-19.

53. Vinciane Despret, Naissance d'une théorie éthologique. La danse du cratérope écaillé, Paris, Les empêcheurs de penser en rond, 1996 ; Vinciane Despret, «La différence comme occasion de pertinence : la question de l'animal », Cahiers de psychologie clinique, №18, 2002, p.11-28 ; Vinciane Despret et Jocelyne Porcher, Être bête, Arles, Actes Sud, 2007. 
En retraçant l'histoire de l'interprétation éthologique des danses des oiseaux du désert du Néguev, V. Despret montre par exemple très précisément comment l'interprétation des différences et des identités est constitutive de l'organisation de nos rapports à nous et aux autres (ici des animaux) : «L'animal offre à l'homme un miroir plus ou moins déformant, plus ou moins acceptable : proche et lointain à la fois, il est à la fois le même et le différent. Le regard que nous portons sur lui, la façon dont nous en construisons la représentation peut le rendre plus "même" ou plus "différent" »; et de s'interroger : "qu'en est-il alors du discours que l'on tient au sujet de ce même-différent? Comment ce discours participe-t-il de cette identité et de cette différence? $\gg^{54}$ On pourrait objecter qu'il ne s'agit là que de discours, mais bon nombre de travaux portant sur les sciences du comportement animal - ceux de V. Despret, de D. Haraway ${ }^{55}$ et bien d'autres encore - montrent précisément comment ces discours produits par les scientifiques sont des propositions ontologiques fortes, faites sur et aux animaux, ne se contentant pas de les qualifier, mais qui les font agir. En un mot, ces travaux prennent au sérieux la capacité transformatrice des sciences et des scientifiques lorsqu'ils produisent des énoncés. Ainsi, ces différences et ces identités perçues, éprouvées, débattues, partagées, ne changent pas seulement notre représentation du monde, elles changent le monde; elles sont ce que V. Despret appelle des «expériences productrices d'existences ${ }^{56}{ }^{5}$. On comprend alors que, non seulement la création d'identité est là aussi pensée comme consubstantielle de la production de différences, mais également que différences et identités «objectives» et «subjectives» sont inextricablement liées dans l'expérience concrète des ajustements anthropozootechniques.

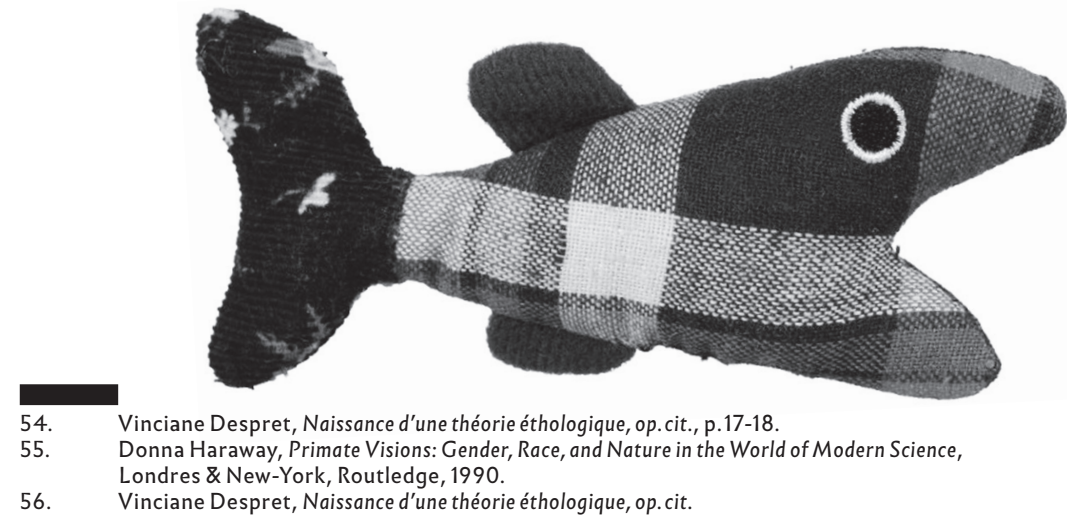


Ainsi, en faisant le bilan de la lecture riche et enrichissante des Fondements oubliés de la culture, plusieurs interrogations apparaissent sur les implications d'un tel programme intégratif et unificateur : quel est finalement le réel gain cognitif du programme proposé, notamment au regard des sacrifices que la mise en ouvre de cette nouvelle synthèse suppose? Nous avons montré qu'en ce qui concerne les sciences sociales, le programme de D. Guillo supposait d'abandonner à la fois des objets, des méthodes mais aussi des données empiriques qui ont permis de développer un savoir conséquent sur les cultures ; ici, les coûts cognitifs paraissent importants au regard des gains (l'exemple de la socialisation de genre en atteste). De plus, on peut estimer qu'il y a un déséquilibre patent dans les efforts demandés aux deux ensembles disciplinaires identifiés : les sacrifices auxquelles les sciences de la nature auraient à consentir semblent en fait assez maigres pour qu'elles puissent s'adapter au nouveau cadre épistémologique visé par D. Guillo. Le sociologue fait ainsi une promotion en demi-teinte de l'apport spécifique des sciences sociales à la compréhension des relations humains-animaux, et par-delà des cultures interspécifiques.

En définitive, c'est bien la dimension totalisante du programme de D. Guillo qui laisse sceptique. En cherchant à proposer une voie unique d'accès (l'interaction) à la totalité de notre compréhension des phénomènes sociaux, D. Guillo laisse entrevoir un monde où les différences entre les disciplines s'estompent, laissant au bord du chemin certaines rées comme incompatibles avec le nouveau paradigme. On ne peut que s'étonner qu'un tenant d'une épistémologie cumulative, construisant un projet empirique centré sur l'attention aux différences, puisse, au nom de l'unité de la science, sacrifier ainsi une telle somme de données et d'approches sur les manières dont se constituent des «nous» et des «eux». Car tel est bien le coût cognitif du biais différentialiste. approches, considé-

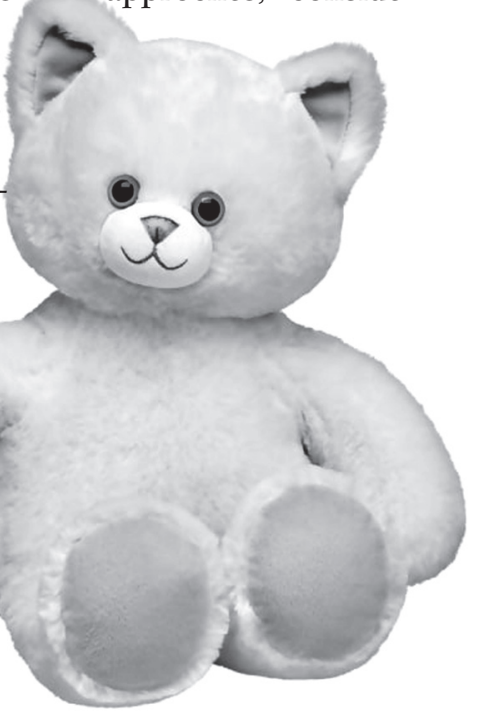

